\title{
Quick Detection of Nodes with Large Degrees
}

\author{
Konstantin Avrachenkov, Nelly Litvak, Marina Sokol, and Don Towsley
}

Abstract. Our goal is to find top- $k$ lists of nodes with the largest degrees in large complex networks quickly. If the adjacency list of the network is known (not often the case in complex networks), a deterministic algorithm to find the top- $k$ list of nodes with the largest degrees requires an average complexity of $\mathcal{O}(n)$, where $n$ is the number of nodes in the network. Even this modest complexity can be very high for large complex networks. We propose to use a random-walk-based method. We show theoretically and by numerical experiments that for large networks, the random-walk method finds good-quality top lists of nodes with high probability and with computational savings of orders of magnitude. We also propose stopping criteria for the random-walk method that requires very little knowledge about the structure of the network.

\section{Introduction}

We are interested in quickly detecting nodes with large degrees in very large networks. Firstly, node degree is one of the centrality measures used for the analysis of complex networks. Secondly, large-degree nodes can serve as proxies for central

Color versions of one or more of the figures in the article can be found online at www.tandfonline.com/uinm.

(C) Taylor \& Francis Group, LLC

ISSN: I542-795I print 
nodes corresponding to other centrality measures such as betweenness centrality and closeness centrality [Lim et al. 11, Maiya and Berger-Wolf 10]. In the present work, we restrict ourselves to undirected networks or symmetrized versions of directed networks. In particular, this assumption is well justified in social networks. Typically, friendship or acquaintance is a symmetric relation. If the adjacency list of the network is known (not often the case in complex networks), a deterministic algorithm to find the top $k$ nodes with the largest degrees requires an average complexity of $\mathcal{O}(n)$, where $n$ is the number of nodes in the network. For instance, if HeapSort is used to find the top $k$ nodes with the largest degrees, the complexity estimate can be specified as $\mathcal{O}(n+k \log (n))$. We assume that the degree is available when a node is accessed (if this is not the case, the complexity should be counted in terms of links). However, even linear complexity can be very high for very large, possibly varying, complex networks. Furthermore, when crawling some online social networks such as Facebook and Twitter, a crawler is constrained by a certain limit on the speed of crawling. For example, Twitter has a limit of one access per minute for the rate of crawling for one standard account. Thus, to crawl the entire network with more than 500 million users, we would need more than 950 years. Certainly, we would like to discover nodes with largest degrees well before the entire network has been crawled.

In the present work, we suggest using random-walk-based methods for detecting a small number of nodes with the largest degrees. The main idea is that the random walk very quickly comes across large-degree nodes. Thus, the analysis of our approach is equivalent to the analysis of hitting times of a random walk. In our numerical experiments, random walks outperform the standard deterministic algorithms by orders of magnitude in terms of computational complexity. For instance, in our experiments with the Web graph of the UK domain (about 18500000 nodes), the random-walk method spends on average only about 5400 steps to detect the largest-degree node. Potential memory savings are also significant, since the method does not require knowledge of the entire network. In many practical applications, we do not need a complete ordering of the nodes and even can tolerate some errors in the top list of nodes. We observe that the randomwalk method obtains many nodes in the top list correctly, and even those nodes that are erroneously placed in the top list have large degrees. Therefore, as typically happens in randomized algorithms [Mitzenmacher and Upfal 05, Motwani and Raghavan 95], we trade off exact results for very good approximate results or for exact results with high probability and gain significantly in computational efficiency.

The paper is organized as follows: In the next section, we introduce our basic random walk with uniform jumps and demonstrate that it is able to find largedegree nodes quickly. Then, in Section 3, using a configuration model, we provide 
an estimate for the necessary number of steps for the random walk. In Section 4, we propose stopping criteria that use very little information about the network. In Section 5, we show the benefits of allowing few erroneous elements in the top- $k$ list. Finally, we conclude the paper in Section 6.

\section{Random Walk with Uniform Jumps}

Let us consider a random walk with uniform jumps that serves as a basic algorithm for quick detection of large-degree nodes. The random walk with uniform jumps is described by the following transition probabilities [Avrachenkov et al. 10a]:

$$
p_{i j}= \begin{cases}\frac{\alpha / n+1}{d_{i}+\alpha}, & \text { if } i \text { has a link to } j, \\ \frac{\alpha / n}{d_{i}+\alpha}, & \text { if } i \text { does not have a link to } j,\end{cases}
$$

where $d_{i}$ is the degree of node $i$. The random walk with uniform jumps can be regarded as a random walk on a modified graph all of whose nodes are connected by artificial edges with weight $\alpha / n$. The parameter $\alpha$ controls the rate of jumps. Introduction of jumps helps in a number of ways. As was shown in [Avrachenkov et al. 10a], it reduces the mixing time to stationarity. It also solves a problem encountered by a random walk on a graph consisting of two or more components, namely the inability to visit all nodes. The random walk with jumps also reduces the variance of the network function estimator [Avrachenkov et al. 10a]. This random walk resembles the PageRank random walk. However, unlike the PageRank random walk, the introduced random walk is reversible. One important consequence of the reversibility of the random walk is that its stationary distribution is given by a simple formula:

$$
\pi_{i}(\alpha)=\frac{d_{i}+\alpha}{2|E|+n \alpha} \quad \forall i \in V
$$

from which the stationary distribution of the unperturbed random walk can easily be retrieved. We observe that the modification preserves the monotonicity of the stationary distribution with respect to the node degree, which is particularly important for our application.

We illustrate on several network examples how the random walk helps us quickly detect large-degree nodes. We consider as examples one synthetic network generated by the preferential attachment rule and two natural large networks. The preferential attachment (PA) network combines 100000 nodes. It was generated according to the generalized preferential attachment mechanism 


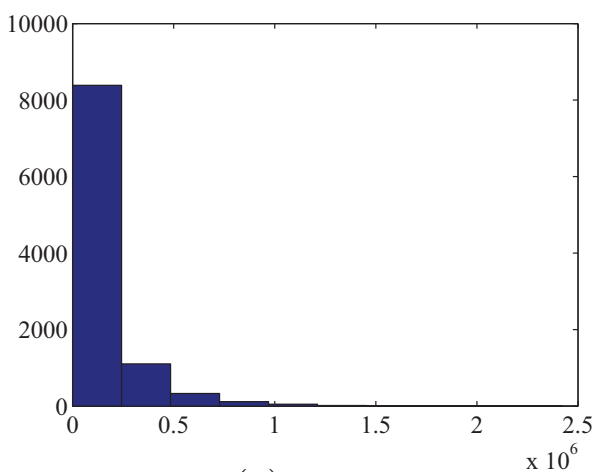

(a) $\alpha=0$

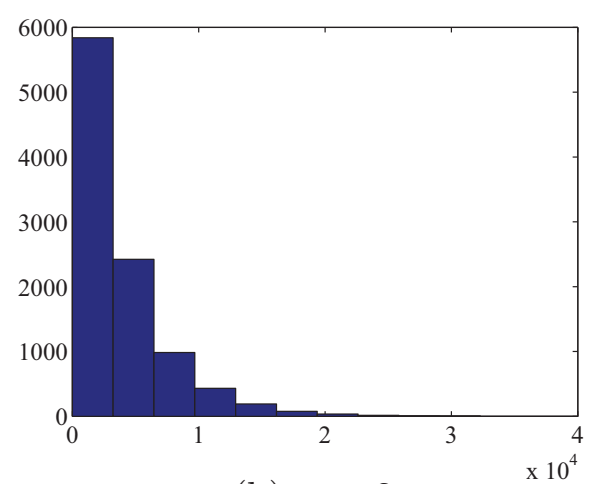

(b) $\alpha=2$

Figure I. Histograms of hitting times in the PA network.

[Dorogovtsev et al. 00]. The average degree of the PA network is 2 , and the power law exponent is 2.5. The first natural example is the symmetrized Web graph of the whole UK domain crawled in 2002 [Boldi and Vigna 04]. The UK network has 18520486 nodes, and its average degree is 28.6. The second natural example is the network of coauthorships of DBLP [Boldi et al. 11]. Each node represents an author, and each link represents a coauthorship of at least one article. The DBLP network has 986324 nodes, and its average degree is 6.8.

We carry out the following experiment: we initialize the random walk (2.1) at a node chosen according to the uniform distribution and continue the random walk until we hit the largest-degree node. The largest degrees for the PA, UK, and DBLP networks are 138, 194955, and 979, respectively. For the PA network, we made 10000 experiments, and for the UK and DBLP networks, we performed 1000 experiments (these networks were too large to perform more).

In Figure 1, we plot histograms of hitting times for the PA network. The first remarkable observation is that when $\alpha=0$ (no restart), the average hitting time, which is equal to 123000 steps, is nearly three orders of magnitude larger than 3720 , the hitting time when $\alpha=2$. The second remarkable observation is that 3720 is of the same order of magnitude as the value

$$
1 / \pi_{\max }(\alpha)=\frac{2|E|+n \alpha}{d_{\max }+\alpha}=2857
$$

which corresponds to the average return time to the largest-degree node in the random walk with jumps.

We were not able to collect a representative number of experiments for the UK and DBLP networks when $\alpha=0$. The reason for this is that the random walk gets 
stuck either in disconnected or weakly connected components of the networks. For the UK network, we were able to make 1000 experiments with $\alpha=0.001$ and obtain the average hitting time 30750 , whereas if we take $\alpha=28.6$ for the UK network, we obtain the average hitting time 5800 . Note that the expected return time to the largest-degree node in the UK network is given by

$$
\frac{1}{\pi_{\max }(\alpha)}=\frac{2|E|+n \alpha}{d_{\max }+\alpha}=5432
$$

For the DBLP graph, we conducted 1000 experiments with $\alpha=0.00001$ and obtained an average hitting time of 41131 , whereas if we take $\alpha=6.8$, we obtain an average hitting time of 14200 . The expected return time to the largest-degree node in the DBLP network is given by

$$
\frac{1}{\pi_{\max }(\alpha)}=\frac{2|E|+n \alpha}{d_{\max }+\alpha}=13607 .
$$

The two natural-network examples confirm our guess that the average hitting time for the largest-degree node is fairly close to the average return time to the largest-degree node, which is reciprocal to the value of the stationary distribution at the largest-degree node. Next, using asymptotic analysis, we show that if $\alpha$ is sufficiently large, the principal term in the asymptotic expansion for the expected hitting time is close to the expected return time. Denote by $H_{j}$ the hitting time to node $j$.

Theorem 2.I. Without loss of generality, index the nodes such that node 1 is a node under consideration, $(1, i) \in E, i=2, \ldots, s, s=d_{1}+1$, and let $\nu$ denote the initial distribution of the random walk with jumps. Then for sufficiently large $\alpha$ and small $\alpha / n$, the expected hitting time to node 1 starting from an arbitrary initial distribution $\nu$ is given by

$$
E_{\nu}\left[H_{1}\right]=\frac{\sum_{i=2}^{n} d_{i}+(n-1) \alpha}{d_{1}+2 \alpha(1-1 / n)}+\mathcal{O}(1) .
$$

Proof. The expected hitting time from distribution $\nu$ to node 1 is given by the formula

$$
E_{\nu}\left[H_{1}\right]=\nu\left[I-P_{-1}\right]^{-1} \underline{1},
$$

where $P_{-1}$ is a taboo probability matrix (i.e., a matrix $P$ with the first row and first column removed). The matrix $P_{-1}$ is substochastic but is very close to stochastic. Let us represent it as a stochastic matrix minus some perturbation 
term:

$$
P_{-1}=\tilde{P}-\varepsilon Q=\tilde{P}-\left[\begin{array}{cccccc}
\frac{1+2 \alpha / n}{d_{2}+\alpha} & 0 & & & 0 \\
0 & \ddots & & & & \\
& & \frac{1+2 \alpha / n}{d_{s}+\alpha} & & & \\
& & & \frac{2 \alpha / n}{d_{s+1}+\alpha} & & \\
& & & & \ddots & 0 \\
& & & & 0 & \frac{2 \alpha / n}{d_{n}+\alpha}
\end{array}\right] \text {. }
$$

We add missing probability mass to the diagonal of $\tilde{P}$, which corresponds to an increase in the weights for self-loops. The matrix $\tilde{P}$ represents a reversible Markov chain with the stationary distribution

$$
\tilde{\pi}_{j}=\frac{d_{j}+\alpha}{\sum_{i=2}^{n} d_{i}+(n-1) \alpha} .
$$

Now we can use the following result from perturbation theory (see [Avrachenkov et al. 10b, Lemma 1]):

$$
[I-\tilde{P}+\varepsilon Q]^{-1}=\frac{\underline{1} \tilde{\pi}}{\tilde{\pi}(\varepsilon Q) \underline{1}}+X_{0}+\varepsilon X_{1}+\cdots
$$

where $\tilde{\pi}$ is the stationary distribution of the stochastic matrix $\tilde{P}$. In our case, the quantity $\max _{i=2, \ldots, s}\left\{1 /\left(d_{i}+\alpha\right), 1 / n\right\}$ will play the role of $\varepsilon$. We apply the series (2.5) to approximate the expected hitting time. Toward this goal, we calculate

$$
\begin{aligned}
\tilde{\pi}(\varepsilon Q) \underline{1} & =\sum_{j=2}^{n} \tilde{\pi}_{j} \varepsilon q_{j j} \\
& =\sum_{j=2}^{s} \frac{d_{j}+\alpha}{\sum_{i=2}^{n} d_{i}+(n-1) \alpha} \frac{1+2 \alpha / n}{d_{j}+\alpha}+\sum_{j=s+1}^{n} \frac{d_{j}+\alpha}{\sum_{i=2}^{n} d_{i}+(n-1) \alpha} \frac{2 \alpha / n}{d_{j}+\alpha} \\
& =\frac{d_{1}(1+2 \alpha / n)+\left(n-d_{1}-1\right)(2 \alpha / n)}{\sum_{i=2}^{n} d_{i}+(n-1) \alpha}=\frac{d_{1}+2 \alpha(1-1 / n)}{\sum_{i=2}^{n} d_{i}+(n-1) \alpha} .
\end{aligned}
$$

Observing that $\nu \underline{1} \tilde{\pi} \underline{1}=1$, we obtain (2.3).

Indeed, the asymptotic expression $(2.3)$ is very close to $(2|E|+n \alpha) /\left(d_{1}+\alpha\right)$, which is the expected return time to node 1 .

Based on the notion of the hitting time, we propose an efficient method for quick detection of the top $k$ largest-degree nodes. The algorithm maintains a top- $k$ candidate list. Note that once one of the $k$ nodes with the largest degrees appears in this candidate list, it remains there subsequently. Thus, we are interested in hitting events. We propose Algorithm 1 for detecting the top $k$ largest-degree nodes. 


\section{Algorithm I. Random walk with jumps and candidate list.}

1. Set $k, \alpha$, and $m$.

2. Execute a random-walk step according to (2.1). If it is the first step, pick the initial node arbitrarily (in particular, the initial node can be chosen by the uniform distribution).

3. Check whether the current node has a larger degree than one of the nodes in the current top- $k$ candidate list. If that is the case, insert the new node in the top- $k$ candidate list and remove the worst node from the list.

4. If the number of random-walk steps is less than $m$, return to step 2 of the algorithm; otherwise, stop.

The value of the parameter $\alpha$ is not crucial. In our experiments, we have observed that as long as the value of $\alpha$ is neither too small nor too big, the algorithm performs well. According to our observations, a good option for the choice of $\alpha$ is a value around the average node degree. Let us explain this choice.

Consider a random walk $\left\{W_{t}\right\}_{t=0}^{\infty}$ with transition probabilities (2.1). We denote by $P_{\nu}(\cdot)$ the probability distribution of this Markov chain with initial distribution $\nu$. Now assume that the Markov chain is in a stationary regime (the stationary regime is achieved quickly when the parameter $\alpha$ is not too small [Avrachenkov et al. 10a]). Then by Bayes's formula, we derive two remarkable equations:

$$
\begin{aligned}
P_{\pi}\left[W_{t}=i \mid \text { jump }\right] & =\frac{P_{\pi}\left[W_{t}=i, \text { jump }\right]}{P_{\pi}[\text { jump }]}=\frac{P_{\pi}\left[W_{t}=i\right] P_{\pi}\left[\text { jump } \mid W_{t}=i\right]}{\sum_{j=1}^{n} P_{\pi}\left[W_{t}=j\right] P_{\pi}\left[\text { jump } \mid W_{t}=j\right]} \\
& =\frac{d_{i}+\alpha}{2|E|+n \alpha} \frac{\alpha}{d_{i}+\alpha} / \sum_{j=1}^{n} \frac{d_{j}+\alpha}{2|E|+n \alpha} \frac{\alpha}{d_{j}+\alpha}=\frac{1}{n},
\end{aligned}
$$

and

$$
\begin{aligned}
P_{\pi}\left[W_{t}=i \mid \text { no jump }\right] & =\frac{P_{\pi}\left[W_{t}=i, \text { no jump }\right]}{P_{\pi}[\text { no jump }]} \\
& =\frac{P_{\pi}\left[W_{t}=i\right] P_{\pi}\left[\text { no jump } \mid W_{t}=i\right]}{\sum_{j=1}^{n} P_{\pi}\left[W_{t}=j\right] P_{\pi}\left[\text { no jump } \mid W_{t}=j\right]} \\
& =\frac{d_{i}+\alpha}{2|E|+n \alpha} \frac{d_{i}}{d_{i}+\alpha} / \sum_{j=1}^{n} \frac{d_{j}+\alpha}{2|E|+n \alpha} \frac{d_{j}}{d_{j}+\alpha} \\
& =\frac{d_{i}}{2|E|}=\pi_{i}(0), \quad i=1,2, \ldots, n .
\end{aligned}
$$


Thus, in a stationary distribution, given that no jump occurred, the probability that $\left[W_{t}=i\right]$ is exactly $\pi_{i}(0)$ !

Next observe that $W_{t}$ is a regenerative process, where regeneration points are the jumps to the uniform distribution, and the regenerating cycles are independent. Concerning the choice of $\alpha$, there is a clear tradeoff: if $\alpha$ is too small, then regenerating cycles are long, and a random walk can get entangled in some part of the network; but if $\alpha$ is too large, then the cycle will often consist of only one step, corresponding to a jump. Thus, we would like to maximize the long-run fraction of independent observations from $\pi(0)$. To this end, we note that given $m^{\prime}$ cycles, the mean total number of steps is

$$
m^{\prime} E[\text { cycle length }]=m^{\prime}\left(P_{\pi}[\text { jump }]\right)^{-1} .
$$

Out of the random walk run with $m^{\prime}$ cycles, $m^{\prime}$ independent observations from $\pi$ are generated, from which on average, $m^{\prime} P_{\pi}$ [jump] observations coincide with a jump. As will be discussed in Section 4, we need to maximize the long-run fraction of independent observations in a sample that are not a jump compared to the number of steps of a random walk:

$$
\frac{m^{\prime}-m^{\prime} P_{\pi}[\text { jump }]}{m^{\prime}\left(P_{\pi}[\text { jump }]\right)^{-1}}=P_{\pi}[\text { jump }]\left(1-P_{\pi}[\text { jump }]\right) \rightarrow \max .
$$

Obviously, the maximum is achieved when

$$
P_{\pi}[\text { jump }]=\frac{1}{2}
$$

It remains to rewrite $P_{\pi}[$ jump] in terms of the algorithm parameters:

$$
\begin{aligned}
P_{\pi}[\text { jump }] & =\sum_{j=1}^{n} P_{\pi}\left[W_{t}=j\right] P_{\pi}\left[\text { jump } \mid W_{t}=j\right] \\
& =\sum_{j=1}^{n} \frac{d_{j}+\alpha}{2|E|+n \alpha} \frac{\alpha}{d_{j}+\alpha}=\frac{n \alpha}{2|E|+n \alpha}=\frac{\alpha}{\bar{d}+\alpha},
\end{aligned}
$$

where $\bar{d}:=2|E| / n$ is the average degree. For maximal efficiency, the last fraction above must be equal to $1 / 2$, which gives the optimal value for the parameter $\alpha$ :

$$
\alpha_{*}=\bar{d}
$$

With this choice of $\alpha$, the random walk contains the greatest possible fraction of independent observations from the distribution $\pi_{i}(0)$.

The average degree is not necessarily known in advance. However, we may choose $\alpha$ based on our knowledge of samples of similar nature and then estimate the average degree using (2.8) and the observed cycle length. Specifically, we can 
use the equation

$$
E_{u}[T]=\frac{1}{P_{\pi}[\mathrm{jump}]}=\frac{2|E| / n+\alpha}{\alpha} .
$$

Then we can adjust $\alpha$ to its optimal value.

Theorem 2.1 demonstrates that the expected hitting time to a large-degree node is approximately equal to the reciprocal of the stationary probability. Next, under technical but natural assumptions, we show that in fact, the reciprocal of the stationary probability is an upper bound on the expected hitting time. Without loss of generality, let us consider node $k$ from the top- $k$ list $\left(d_{1} \geq \cdots \geq\right.$ $\left.d_{k} \geq d_{k+1} \geq \cdots\right)$. Assume also that the initial node is chosen according to the uniform distribution. Let $H_{k}$ be the hitting time to node $k$, and let $T$ be the time of the first jump (to the uniform distribution). Then, using Wald's identity, we can write

$$
E_{u}\left[H_{k}\right]=E_{u}\left[\# \text { jumps on }\left[0, H_{k}\right]\right] E_{u}\left[\min \left\{T, H_{k}\right\}\right],
$$

where $E_{u}[\cdot]$ is the expectation given that the random walk starts from the uniform distribution. We note that

$$
E_{u}\left[\min \left\{T, H_{k}\right\}\right] \leq E_{u}[T]
$$

We also note that

$$
E_{u}\left[\# \text { jumps on }\left[0, H_{k}\right]\right]=\frac{1}{P_{u}\left[H_{k} \leq T\right]} .
$$

Next, we provide a lower bound for the probability $P_{u}\left[H_{k} \leq T\right]$. This lower bound gives a good approximation, if we assume that node $k$ is usually found within the first two steps of a cycle. This is a natural assumption, if $\alpha$ is not too small and, consequently, the cycles are not too large. In particular, this is the case if we choose the value of $\alpha$ as the average degree. Then we have

$$
\begin{aligned}
P_{u}\left[H_{k} \leq T\right] & \geq P_{u}\left[H_{k} \leq \min \{T, 2\}\right]=\frac{1}{n}+\frac{1}{n} \sum_{i:(i, k) \in E} \frac{\alpha / n+1}{d_{i}+\alpha} \\
& >\frac{d_{k}}{n} \cdot \frac{1}{d_{k}} \sum_{i:(i, k) \in E} \frac{\alpha / n+1}{d_{i}+\alpha} \geq \frac{d_{k}}{n} \cdot \frac{\alpha / n+1}{d_{k}^{-1} \sum_{i:(i, k) \in E} d_{i}+\alpha} .
\end{aligned}
$$

Combining the above equation with (2.10)-(2.13), we obtain

$$
E_{u}\left[H_{k}\right] \leq \frac{n}{d_{k}} \cdot \frac{\bar{d}+\alpha}{\alpha} \cdot \frac{d_{k}^{-1} \sum_{i:(i, k) \in E} d_{i}+\alpha}{\alpha / n+1}
$$


In particular, choosing $\alpha=\bar{d}$ in (2.15) yields

$$
E_{u}\left[H_{k}\right] \leq \frac{2 n}{d_{k}} \cdot \frac{d_{k}^{-1} \sum_{i:(i, k) \in E} d_{i}+\bar{d}}{\bar{d} / n+1} .
$$

The number $m$ of random-walk steps is a crucial parameter. Our experiments indicate that we obtain a top- $k$ list with many correct elements with high probability if we take the number of random-walk steps to be two or three times as large as the expected hitting time of the nodes in the top- $k$ list. This observation can be made rigorous thanks to a result from [Bollobás 98, p. 333], which we can adapt for our situation as follows.

Proposition 2.2. Let $H_{1}, \ldots, H_{k}$ denote the hitting times to the top $k$ nodes with the largest degrees $\left(d_{1} \geq \cdots \geq d_{k} \geq d_{k+1} \geq \cdots\right)$. Then the expected time $E_{u}[\tilde{H}]$ for the random walk with transition probabilities (2.1) starting from the uniform distribution to detect a fraction $\beta$ of top- $k$ nodes is bounded by

$$
E_{u}[\tilde{H}] \leq \frac{1}{1-\beta} E_{u}\left[H_{k}\right] .
$$

From Theorem 2.1 or bound (2.16), we know that the expected hitting time of a large-degree node is related to the value of the node's degree. Thus, the problem of choosing $m$ reduces to the problem of estimating the values of the largest degrees. We address this problem in the following section.

\section{Estimating the Largest Degrees in the Configuration Network Model}

The estimates of the values of the largest degrees can be derived in the configuration network model [van der Hofstad 09] with a power-law degree distribution. In some applications, the knowledge of the power-law parameters might be available to us. For instance, it is known that Web graphs have power-law degree distribution, and we know typical ranges for the power-law parameters (see, e.g., [Barabási and Albert 99]).

We assume that the node degrees $D_{1}, \ldots, D_{n}$ are i.i.d. random variables with a power-law distribution $F$ and finite expectation $E[D]$. Let us determine the number of links contained in the top $k$ nodes. Define

$$
F(x)=P[D \leq x], \quad \bar{F}(x)=1-F(x), \quad x \geq 0 .
$$

Further, let $D_{(1)} \geq \cdots \geq D_{(n)}$ be the order statistics of $D_{1}, \ldots, D_{n}$. Under the assumption that the $D_{j}$ obey a power law, we use the results from the extreme 
value theory as presented in [Matthys and Beirlant 03] to state that there exist sequences of constants $\left(a_{n}\right)$ and $\left(b_{n}\right)$ and a constant $\delta$ such that

$$
\lim _{n \rightarrow \infty} n \bar{F}\left(a_{n} x+b_{n}\right)=(1+\delta x)^{-1 / \delta} .
$$

This implies the following approximation for high quantiles of $F$, with exceedance probability close to zero [Matthys and Beirlant 03]:

$$
x_{p} \approx a_{n} \frac{(p n)^{-\delta}-1}{\delta}+b_{n} .
$$

For the $j$ th-largest degree, where $j=2, \ldots, k$, the estimated exceedance probability equals $(j-1) / n$, and thus we can use the quantile $x_{(j-1) / n}$ to approximate the degree $D_{(j)}$ of this node:

$$
D_{(j)} \approx a_{n} \frac{(j-1)^{-\delta}-1}{\delta}+b_{n} .
$$

The sequences $\left(a_{n}\right)$ and $\left(b_{n}\right)$ are easy to find for a given shape of the tail of $F$. Below, we derive the corresponding results for the commonly accepted Pareto tail distribution of $D$, that is,

$$
\bar{F}(x)=C x^{-\gamma} \text { for } x>x^{\prime},
$$

where $\gamma>1$ and $x^{\prime}$ is a fixed sufficiently large number, so that the power-law degree distribution is observed for nodes with degree larger than $x^{\prime}$. In that case, we have

$$
\begin{aligned}
\lim _{n \rightarrow \infty} n \bar{F}\left(a_{n} x+b_{n}\right) & =\lim _{n \rightarrow \infty} n C\left(a_{n} x+b_{n}\right)^{-\gamma} \\
& =\lim _{n \rightarrow \infty}\left(C^{-1 / \gamma} n^{-1 / \gamma} a_{n} x+C^{-1 / \gamma} n^{-1 / \gamma} b_{n}\right)^{-\gamma},
\end{aligned}
$$

which directly gives (3.1) with

$$
\delta=1 / \gamma, \quad a_{n}=\delta C^{\delta} n^{\delta}, \quad b_{n}=C^{\delta} n^{\delta} .
$$

Substituting (3.4) into (3.2), we obtain the following prediction for $D_{(j)}, j=$ $2, \ldots, k$, in the case of the Pareto tail of the degree distribution:

$$
D_{(j)} \approx C^{1 / \gamma}(j-1)^{-1 / \gamma} n^{1 / \gamma} .
$$

It remains to find an approximation for $D_{(1)}$, the maximal degree in the graph. From the extreme value theory, it is well known that if $D_{1}, \ldots, D_{n}$ obey a power law, then

$$
\lim _{n \rightarrow \infty} P\left(\frac{D_{(1)}-b_{n}}{a_{n}} \leq x\right)=H_{\delta}(x)=\exp \left(-(1+\delta x)^{-1 / \delta}\right),
$$

where for the Pareto tail, $a_{n}, b_{n}$, and $\delta$ are defined in (3.4). Thus, as an approximation for the maximal node degree, we can choose $a_{n} x+b_{n}$, where $x$ can be 
chosen as either a mean, a median, or a mode of $H_{\delta}(x)$. If we choose the mode $\left((1+\delta)^{-\delta}-1\right) / \delta$, then we obtain an approximation that is smaller than that for the second-largest degree. Further, the mean $(\Gamma(1-\delta)-1) / \delta$ is very sensitive to the value of $\delta=1 / \gamma$, especially when $\gamma$ is close to 1 , which is often the case in complex networks. Besides, the parameter $\gamma$ is hard to estimate with high precision. Thus, we suggest choosing the median $\left.(\log (2))^{-\delta}-1\right) / \delta$, which is less sensitive to the value of $\delta$. This yields

$$
D_{(1)} \approx a_{n} \frac{(\log (2))^{-\delta}-1}{\delta}+b_{n}=C^{1 / \gamma}(\log (2))^{-1 / \gamma} n^{1 / \gamma}
$$

For instance, in the PA network, $\gamma=2.5$ and $C=3.7$, which gives, according to $(3.6), D_{(1)} \approx 195$. (This is a reasonably good prediction, even though the PA network is not generated according to the configuration model. We also note that even though the extremum distribution in the preferential attachment model is different from that of the configuration model, their ranges seem to be quite close [Moreira et al. 02].) This, in turn, suggests that for the PA network, $m$ should be chosen in the range $6000-18000$ if $\alpha=2$. As we can see from Figure 2, this is indeed a good range for the number of random-walk steps. In the UK network, $\gamma=1.7$ and $C=90$, which gives $D_{(1)} \approx 329820$ and suggests a range of 20000 30000 for $m$ if $\alpha=28.6$. Figure 3 confirms that this is a good choice. The degree distribution of the DBLP network does not follow a power law, so we cannot apply the above reasoning to it.

We conclude this section with a remark that follows from equation (3.5), bound (2.16), and Proposition 2.2. It then follows that we can find a $\beta$ fraction of top- $k$ largest-degree nodes in sublinear expected time in the configuration model. That is, we have

$$
E_{u}[\tilde{H}] \leq \frac{2}{1-\beta} \cdot \frac{d_{k}^{-1} \sum_{i:(i, k) \in E} d_{i}+\bar{d}}{\bar{d} / n+1} \cdot \frac{n}{C^{1 / \gamma}(k-1)^{-1 / \gamma} n^{1 / \gamma}}
$$

In particular, the last fraction above is of the order $\tilde{C} n^{\frac{\gamma-1}{\gamma}}$. If $\gamma$ is close to one (which is often the case in complex networks), the computational savings compared to the deterministic approach can be very significant. For instance, for the UK network with $k=10$ and $\beta=0.8$, the bound (2.17) gives

$$
E_{u}[\tilde{H}] \leq 214150
$$

which means at least 86-fold computational savings. 


\section{Stopping Criteria}

Suppose now that we do not have any information about the range for the $k$ largest degrees. In this section, we design stopping criteria that do not require knowledge about the structure of the network. As we shall see, knowledge of the order of magnitude of the average degree might help, but this knowledge is not imperative for a practical implementation of the algorithm.

Let us now assume that node $j$ can be sampled independently with probability $\pi_{j}(\alpha)$ as in (2.2). There are at least two ways to achieve this practically. The first approach is to run the random walk for a significant number of steps until it reaches the stationary distribution. If one chooses $\alpha$ reasonably large, say the same order of magnitude as the average degree, then the mixing time becomes quite small [Avrachenkov et al. 10a], and we can be sure to reach the stationary distribution in a small number of steps. Then, the last step of a run of the random walk will produce an i.i.d. sample from a distribution very close to (2.2). The second approach is to run the random walk uninterruptedly, also with a significant value of $\alpha$, and then perform Bernoulli sampling with probability $q$ after a small initial transient phase. If $q$ is not too large, we shall have nearly independent samples following the stationary distribution (2.2). In our experiment, $q \in[0.2,0.5]$ gives good results when $\alpha$ has the same order of magnitude as the average degree.

We now estimate the probability of detecting correctly the top $k$ nodes after $m$ i.i.d. samples from (2.2). Denote by $X_{i}$ the number of hits at node $i$ after $m$ i.i.d. samples. We note that if we use the second approach to generate i.i.d. samples, we spend approximately $m / q$ steps of the random walk. We correctly detect the top- $k$ list with the probability given by the multinomial distribution

$$
\begin{aligned}
P & {\left[X_{1} \geq 1, \ldots, X_{k} \geq 1\right] } \\
& =\sum_{i_{1} \geq 1, \ldots, i_{k} \geq 1} \frac{m !}{i_{1} ! \cdots i_{k} !\left(m-i_{1}-\cdots-i_{k}\right) !} \pi_{1}^{i_{1}} \cdots \pi_{k}^{i_{k}}\left(1-\sum_{i=1}^{k} \pi_{i}\right)^{m-i_{1}-\cdots-i_{k}}
\end{aligned}
$$

but it is infeasible for any realistic computations. Therefore, we propose to use the Poisson approximation. Let $Y_{j}, j=1, \ldots, n$, be independent Poisson random variables with means $\pi_{j} m$. That is, the random variable $Y_{j}$ has the following probability mass function: $P\left[Y_{j}=r\right]=e^{-m \pi_{j}}\left(m \pi_{j}\right)^{r} / r$ !. It is convenient to work with the complementary event of not detecting correctly the top- $k$ list. Then we 
have

$$
\begin{aligned}
& P\left[\left\{X_{1}=0\right\} \cup \cdots \cup\left\{X_{k}=0\right\}\right] \leq 2 P\left[\left\{Y_{1}=0\right\} \cup \cdots \cup\left\{Y_{k}=0\right\}\right] \\
& \quad=2\left(1-P\left[\left\{Y_{1} \geq 1\right\} \cap \cdots \cap\left\{Y_{k} \geq 1\right\}\right]\right) \\
& \quad=2\left(1-\prod_{j=1}^{k} P\left[\left\{Y_{j} \geq 1\right\}\right]\right) \\
& \quad=2\left(1-\prod_{j=1}^{k}\left(1-P\left[\left\{Y_{j}=0\right\}\right]\right)\right)=2\left(1-\prod_{j=1}^{k}\left(1-e^{-m \pi_{j}}\right)\right)=: a
\end{aligned}
$$

where the first inequality follows from [Mitzenmacher and Upfal 05, Theorem 5.10]. In fact, in our numerical experiments, we observed that the factor 2 in the first inequality is very conservative. For large values of $m$, the Poisson bound without 2 works very well as a proper approximation.

For example, if we would like to obtain the top-10 list with at most $10 \%$ probability of error, we need to have on average 4.5 hits for each top element. This can be used to design the stopping criteria for our random-walk algorithm. Let $\bar{a} \in(0,1)$ be the admissible probability of an error in the top- $k$ list. The idea now is to stop the algorithm after $m$ steps when the estimated value of $a$ for the first time is lower than the critical number $\bar{a}$. Clearly,

$$
\hat{a}_{m}=2\left(1-\prod_{j=1}^{k}\left(1-e^{-X_{j}}\right)\right)
$$

is the maximum likelihood estimator for $a$, so we would like to choose $m$ such that $\hat{a}_{m} \leq \bar{a}$. The problem, however, is that we do not know which $X_{j}$ 's are the realizations of the number of visits to the top $k$ nodes. Then let $X_{j_{1}}, \ldots, X_{j_{k}}$ be the number of hits to the current elements in the top- $k$ candidate list and consider the estimator

$$
\hat{a}_{m, 0}=2\left(1-\prod_{i=1}^{k}\left(1-e^{-X_{j_{i}}}\right)\right),
$$

which is the maximum likelihood estimator of the quantity

$$
2\left(1-\prod_{i=1}^{k}\left(1-e^{-m \pi_{j_{i}}}\right)\right) \geq a .
$$

(Here $\pi_{j_{i}}$ is a stationary probability of the node with the score $X_{j_{i}}, i=1, \ldots, k$.) The estimator $\hat{a}_{m, 0}$ is computed without knowledge of the top $k$ nodes or their 
degrees, and it is an estimator of an upper bound of the estimated probability that there are errors in the top- $k$ list. This leads to the following stopping rule.

Stopping Rule 4.I. Stop at $m=m_{0}$, where

$$
m_{0}=\arg \min \left\{m: \hat{a}_{m, 0} \leq \bar{a}\right\} .
$$

The above stopping criterion can be simplified even further to avoid computation of $\hat{a}_{m, 0}$. Since

$$
\hat{a}_{m, 1}:=2\left(1-\left(1-e^{-X_{j_{k}}}\right)^{k}\right) \geq \hat{a}_{m, 0} \geq \hat{a},
$$

where $X_{j_{k}}$ is the number of hits of the worst element in the candidate list, the inequality $\hat{a}_{m} \leq \bar{a}$ is guaranteed if $\hat{a}_{m, 1} \leq \bar{a}$. This leads to the following stopping rule for the random-walk algorithm.

Stopping Rule 4.2. Compute $x_{0}=\arg \min \left\{x \in \mathbb{N}:\left(1-e^{-x}\right)^{k} \geq 1-\bar{a} / 2\right\}$. Stop at

$$
m_{1}=\arg \min \left\{m: X_{j_{k}}=x_{0}\right\}
$$

We have observed in our numerical experiments that we obtain the best tradeoff between the number of steps of the random walk and the accuracy if we take $\alpha$ around the average degree and the sampling probability $q$ around 0.5 . Specifically, if we take $\bar{a} / 2=0.15\left(x_{0}=4\right)$ in Stopping Rule 4.2 for the top-10 list, we obtain $87 \%$ accuracy for an average of 47000 random-walk steps for the PA network, $92 \%$ accuracy for an average of 174468 random-walk steps for the DBLP network, and $94 \%$ accuracy for an average of 247166 random-walk steps for the UK network. We have averaged over 1000 experiments to obtain tight confidence intervals.

\section{Relaxation of Top-k Lists}

In the stopping criteria of the previous section, we attempted to detect all nodes in the top- $k$ list. This costs us a considerable numbers of steps of the random walk. We can significantly gain in performance by relaxing this strict requirement. For instance, we could just ask for list of $k$ nodes that contains $80 \%$ of 
the top $k$ nodes [Avrachenkov et al. 11]. In this way, we can take advantage of a generic $80 / 20$ rule that $80 \%$ of the result can be achieved with $20 \%$ of the effort.

Let us calculate the expected number of the top $k$ elements observed in the candidate list up to trial $m$. Define by $X_{j}$ the number of times we have observed node $j$ after $m$ trials and

$$
H_{j}= \begin{cases}1, & \text { if node } j \text { has been observed at least once, } \\ 0, & \text { if node } j \text { has not been observed. }\end{cases}
$$

Assuming that we sample in i.i.d. fashion from the distribution (2.2), we can write

$$
\begin{aligned}
E\left[\sum_{j=1}^{k} H_{j}\right] & =\sum_{j=1}^{k} E\left[H_{j}\right]=\sum_{j=1}^{k} P\left[X_{j} \geq 1\right]=\sum_{j=1}^{k}\left(1-P\left[X_{j}=0\right]\right) \\
& =\sum_{j=1}^{k}\left(1-\left(1-\pi_{j}\right)^{m}\right) .
\end{aligned}
$$

In Figure 2, we plot $E\left[\sum_{j=1}^{k} H_{j}\right]$ (the curve "I.I.D. sample") as a function of $m$ for $k=10$ for the PA network with $\alpha=0$ and $\alpha=2$. In Figure 3, we plot $E\left[\sum_{j=1}^{k} H_{j}\right]$ as a function of $m$ for $k=10$ for the UK network with $\alpha=0.001$ and $\alpha=28.6$. The results for the UK and DBLP networks are similar in spirit.

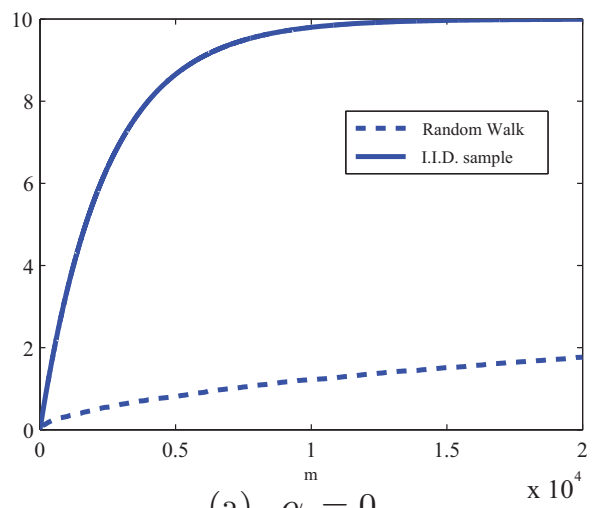

(a) $\alpha=0$

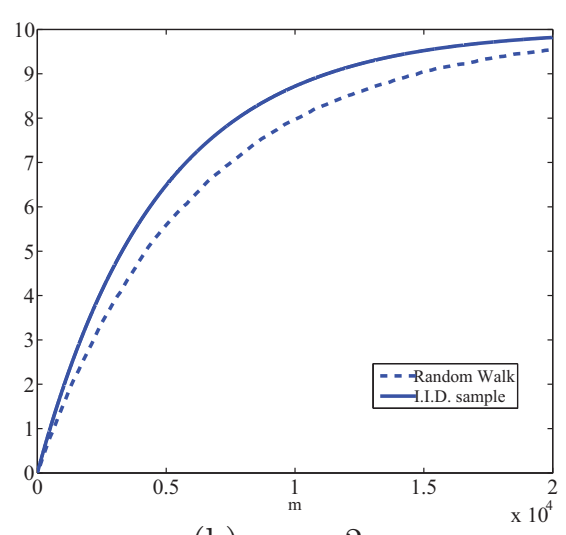

(b) $\alpha=2$

Figure 2. Average number of correctly detected elements in top 10 for PA. 


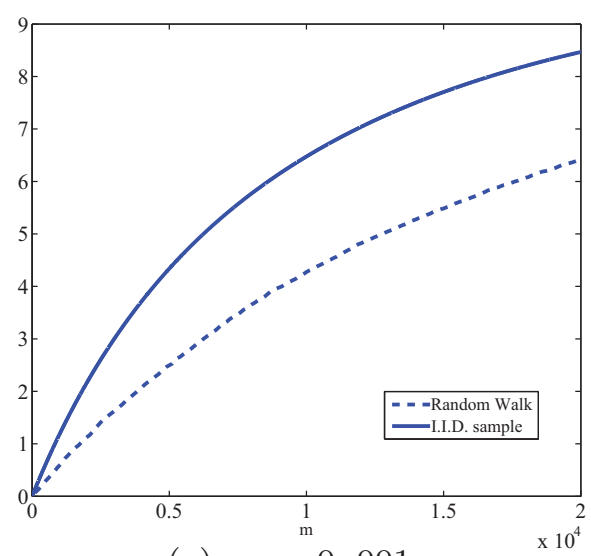

(a) $\alpha=0.001$

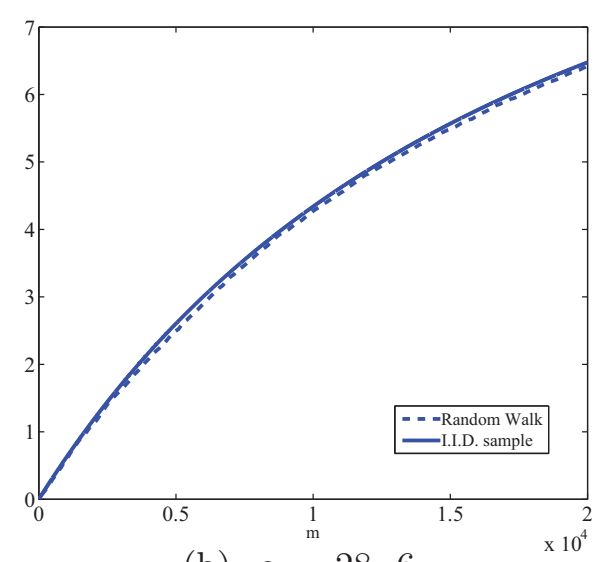

(b) $\alpha=28.6$

Figure 3. Average number of correctly detected elements in top 10 for UK.

Here again we can use the Poisson approximation

$$
E\left[\sum_{j=1}^{k} H_{j}\right] \approx \sum_{j=1}^{k}\left(1-e^{-m \pi_{j}}\right) .
$$

In fact, the Poisson approximation is so good that if we plot it in Figures 2 and 3 , it nearly covers exactly the curves labeled "I.I.D. sample," which correspond to the exact formula (5.1). Similarly to the previous section, we can propose stopping criteria based on the Poisson approximation. Set

$$
b_{m}=\sum_{i=1}^{k}\left(1-e^{-X_{j_{i}}}\right) .
$$

Stopping Rule 5.I. Stop at $m=m_{2}$, where

$$
m_{2}=\arg \min \left\{m: b_{m} \geq \bar{b}\right\} \text {. }
$$

Now if we take $\bar{b}=7$ in Stopping Rule 5.1 for the top-10 list, we obtain, on average, 8.89 correct elements for an average of 16725 random-walk steps for the PA network; we obtain, on average, 9.28 correct elements for an average of 66860 random-walk steps for the DBLP network; and we obtain, on average, 9.22 correct elements for an average of 65802 random-walk steps for the UK network. (We have averaged over 1000 experiments for each network.) This represents for 
the UK network a gain of more than two orders of magnitude in computational complexity with respect to the deterministic algorithm.

\section{Conclusions and Future Research}

We have proposed the random-walk method with the candidate list for quick detection of largest-degree nodes and analyzed the complexity of the method by means of random-walk hitting times. We have also supplied stopping criteria that do not require knowledge of the graph structure. In the case of large networks, our algorithm finds the top- $k$ list of largest-degree nodes with few mistakes with running time orders of magnitude faster than deterministic algorithms. In future research, we plan to obtain estimates of the required number of steps for various types of complex networks and to design methods for directed networks. In particular, it would be of interest to analyze in greater detail how assortativity and clustering of networks affect the performance of the method.

Acknowledgments. We would like to thank Ali Eshragh for helpful remarks that we received during the preparation of the manuscript.

Funding. This research was sponsored by INRIA Alcatel-Lucent Joint Lab, by the European Commission within the framework of the CONGAS project FP7-ICT-2011-8317672, by EU-FET Open Grant NADINE (288956), by the NSF under CNS-1065133, and the U.S. Army Research Laboratory under Cooperative Agreement W911NF-09$2-0053$.

\section{References}

[Avrachenkov et al. 10a] K. Avrachenkov, B. Ribeiro, and D. Towsley. "Improving Random Walk Estimation Accuracy with Uniform Restarts." In Proceedings of WAW 2010, LNCS 6516, pp. 98-109. Springer, 2010.

[Avrachenkov et al. 10b] K. Avrachenkov, V. Borkar, and D. Nemirovsky. "Quasistationary Distributions as Centrality Measures for the Giant Strongly Connected Component of a Reducible Graph." Journal of Comp. and Appl. Mathematics 234 (2010), 3075-3090.

[Avrachenkov et al. 11] K. Avrachenkov, N. Litvak, D. Nemirovsky, E. Smirnova, and M. Sokol. "Quick Detection of Top- $k$ Personalized PageRank Lists." In Proceedings of WAW 2011, pp. 50-61, 2011.

[Barabási and Albert 99] A. L. Barabási and R. Albert. "Emergence of Scaling in Random Networks." Science 286 (1999), 509-512.

[Boldi and Vigna 04] P. Boldi and S. Vigna. "The WebGraph Framework I: Compression Techniques." in Proceedings of $W W W$ 2004, pp. 595-602, 2004. 
[Boldi et al. 11] P. Boldi, M. Rosa, M. Santini, and S. Vigna. "Layered Label Propagation: A Multiresolution Coordinate-Free Ordering for Compressing Social Networks." In Proceedings of $W W W$ 2011, pp. 587-596, 2011.

[Bollobás 98] B. Bollobás. Modern Graph Theory. Springer, 1998.

[Dorogovtsev et al. 00] S. N. Dorogovtsev, J. F. F. Mendes, and A. N. Samukhin. "Structure of Growing Networks: Exact Solution Of The Barabási-Albert Model." Phys. Rev. Lett. 85 (2000), 4633-4636.

[van der Hofstad 09] R. van der Hofstad. "Random Graphs and Complex Networks." Available online http://www.win.tue.nl/rhofstad/NotesRGCN.pdf, 2009.

[Lim et al. 11] Y. Lim, D. S. Menasche, B. Ribeiro, D. Towsley, and P. Basu. "Online Estimating the $k$ Central Nodes of a Network." In Proceedings of IEEE NSW 2011, pp. 118-122, 2011.

[Maiya and Berger-Wolf 10] A. S. Maiya and T. Y. Berger-Wolf. "Online Sampling of High Centrality Individuals in Social Networks." In Proceedings of PAKDD 2010, pp. 91-98, 2010.

[Matthys and Beirlant 03] G. Matthys and J. Beirlant. "Estimating the Extreme Value Index and High Quantiles with Exponential Regression Models." Statistica Sinica 13 (2003), 853-880.

[Mitzenmacher and Upfal 05] M. Mitzenmacher and E. Upfal. Probability and Computing: Randomized Algorithms and Probabilistic Analysis. Cambridge University Press, 2005.

[Moreira et al. 02] A. A. Moreira, J. S. Andrade Jr., and L. A. N. Amaral. "Extremum Statistics in Scale-Free Network Models." Phys. Rev. Lett. 89 (2002), 268703.

[Motwani and Raghavan 95] R. Motwani and P. Raghavan. Randomized Algorithms. Cambridge University Press, 1995.

Konstantin Avrachenkov, Inria Sophia Antipolis, 2004 Route des Lucioles, 06902 Sophia Antipolis, France (k.avrachenkov@sophia.inria.fr)

Nelly Litvak, University of Twente, Faculty of Electrical Engineering, Mathematics and Computer Sciences, Stochastic Operational Research Department, P.O. Box 217, 7500 AE Enschede, The Netherlands (n.litvak@utwente.nl)

Marina Sokol, Inria Sophia Antipolis, 2004 Route des Lucioles, 06902 Sophia Antipolis, France (marina.sokol@sophia.inria.fr)

Don Towsley, University of Massachusetts, Department of Computer Science, Amherst, MA 01003, USA (towsley@cs.umass.edu) 